\title{
Communication Antenas for UAVs
}

\author{
Pedro Marques ${ }^{1}$, Maria Martins ${ }^{1}$, António Baptista ${ }^{2}$ and João Paulo N. Torres ${ }^{3, *}$ \\ ${ }^{I}$ CINAMIL, Centro de Investigação da Academia Militar, Academia Militar, Lisboa, Portugal \\ ${ }^{2}$ Departamento de Engenharia Electrotécnica, Instituto Superior Técnico, Lisboa Portugal \\ ${ }^{3}$ Instituto de Telecomunicações, Departamento de Engenharia Electrotécnica, Instituto Superior Técnico, Lisboa Portugal
}

Received 10 August 2017; Accepted 14 November 2017

\begin{abstract}
Over the past decades, particularly the last, the use of unmanned aerial platforms known as Unmanned Air Vehicle (UAV) by the military has played a key role in the conduct of operations, whether they be at a military or civilian level. The Portuguese Air Force similar to what happens with other NATO counterparts is developing a project called "Project for Research and Technology in Unmanned Aerial Vehicles" (PIVANT) with the aim of providing the Portuguese Air Force with the ability of exploitation of these unmanned aerial vehicles, having already developed some UAV platforms running reconnaissance and surveillance missions over continental and maritime Portuguese territory. The need to communicate or improve communication with these platforms has led to the proposal research, whose goal is to design an antenna that enables improved efficiency and scope of the downlink between the UAV and the ground station. In this regard an Electronically Steerable Parasitic Array antenna radiator (ESPAR) will be designed and simulated. This antenna allows the variation of the direction of maximum gain in azimuth, which will give the operator the possibility to choose the intended direction of radiation. The antenna will be designed and simulated using the CST MWS program, to operate in the band of $1.33 \mathrm{GHz}$. After this stage, the antenna will be constructed and implemented in the UAV to carry out tests.
\end{abstract}

Keywords: ESPAR, antenna, communication, gain direction, UAV

\section{Introduction}

In current operations theatres (OT), information plays a key role in the conduction of military actions, having superiority the force that more effectively and rapidly acquires this information, preventing the opponent to react in a timely manner. One of the technological tools that has increased the effectiveness of military operations is the Unmanned Air Vehicle (UAV) [1].

In this regard the use of UAVs in the operations theatre is a tool with increasingly importance in the conduct of military operations, specially surveillance and reconnaissance missions, since it offers timely information in any environment without the use of conventional forces.

The Portuguese Army and the Portuguese Air Force are developing platforms for running such missions. The Army with the acquisition of surveillance robots under the ROVIM project and the Air Force with the development of UAVs under the PIVANT project.

This is the rationale and background for the present research which includes theoretical analysis, design and simulation of a directional planar antenna operating in the band $1.33 \mathrm{GHz}$, with the configuration of an Electronically Steerable Parasitic Array Radiator (ESPAR) antenna.

The antenna will be placed in the UAV wing, not only to ensure the connection of data, including video, but also to

\footnotetext{
*E-mail address: jocotorres@ist.utl.pt

ISSN: 1791-2377 @ 2018 Eastern Macedonia and Thrace Institute of Technology. All rights reserved. doi:10.25103/jestr.111.11
}

improve and increase the UAV communication distance with the ground station. The planar antenna ESPAR (P-ESPAR) should allow azimuthal variation of the radiation pattern of the main lobe. The antenna has to comply with the operating requirements, namely bandwidth, gain and SWR (standing wave ratio) ensuring UAV continuous communication with the ground station. In addition to these operational features, the antenna must have reduced dimensions and be robust.

The simulation of the antenna radiation parameters is performed using an appropriate simulation program CST Microwave Studio (CST MWS). The design or layout for future construction will be done in 3D CAD Solidworks design program.

\section{State of the Art of the UAV Antennas}

\subsection{Communication, Communication means and Frequencies}

The communication between the ground station (GS) and the UAV is based on two transmission links, one upwards and the other downwards, called "up-link" and "down-link". The "up-link" is including the command and control transmission from operators to UAVs, including flight trajectories (stored on autopilot controller or Autonomous flight control system (AFCS), payload and information of the current position of the ground station when required. In the descending link, corresponding to the "down-link", is ensured transmission of data collected as images and videos, the current position of the UAV and other aircraft operating information (fuel, etc.) to the ground station, usually by a directive antenna. 
In this sense, the maintenance of communications is extremely important in the operations performed by UAVs.

Shannon [2] deepened Nyquist results and proved that the maximum capacity $C$ of a channel [bits / s] is given by the following equation:

$$
C=L B g_{2}(1+S N R)
$$

In which $L B$ is the bandwidth in $\mathrm{Hz}$ and $(S N R)$ is the signal to noise ratio in $\mathrm{dB}$. Through the above equation it can be seen that the channel capacity is directly proportional to the bandwidth, which in turn depends on the frequency, so for high frequencies the transmission capacity will be higher, indispensable component for the UAV to collect and share information with the ground station. In addition, the use of these frequencies requires line of sight between the receiving and transmitting antennas [2][3].

\subsection{Antenna types used by UAVs}

The omnidirectional antennas provide a radiation pattern in the shape of a $360^{\circ}$ donut [4]. These types of antennas used in UAVs are generally constituted only by a vertical monopole with a length corresponding to quarter of the operating wavelength $1 / 4 \lambda$. This antenna is vertically polarized and requires a receiving antenna with similar polarization. The monopole antenna radiation diagram is omnidirectional and the received power decreases rapidly with distance [5]. In figure (1) (a) we may observe that this type of antenna is normally placed at the bottom front of the UAV.

Consequently to obtain higher gains and more directive radiation patterns, antenna array are used. The individual fields originated by the antennas interfere with each other, creating maximum and null radiation in specific directions [6] [7](fig.1).

Currently the antennas arrays more used in UAVs are linear arrays of dipoles and planar antennas arrays. This type of antenna provides a better SNR, greater directivity, higher gain and may also have the ability to direct the antenna radiation for a given desired direction [3]. For aerodynamic reasons these antennas should be implemented on wings of UAVs, reducing air resistance generated during flight due to drag effects, improving the performance of the UAV [2].
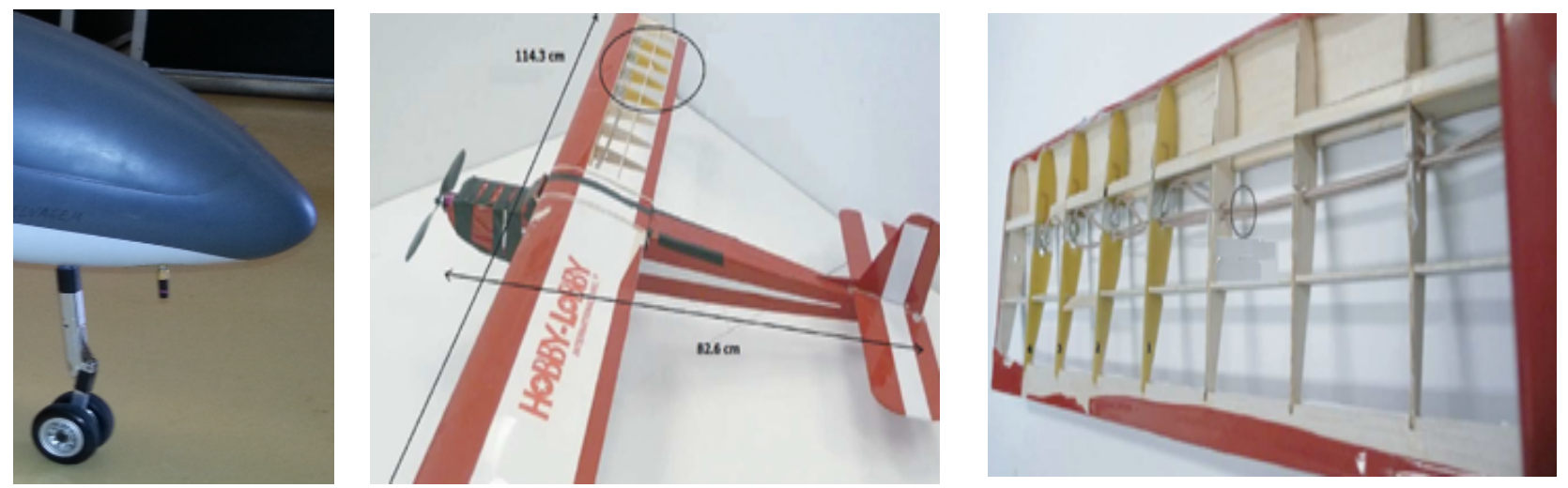

Fig. 1. a) Monopole $1 / 4 \lambda$ implemented in UAV; b) UAV model using a linear array of antennas embedded in the wing structure [8] c) Overview of the antenna embedded in the wing UAV [8]

Antennas have been following the trend towards miniaturization and integration with the intended application.

In UAVs communication these factors are of extreme importance, hence the reason for the great applicability of planar antennas in UAVs, which present certain advantages over the conventional ones, namely:

- $\quad$ reduced volume and lighter structures;

- $\quad$ easy to adapt to different surfaces for mounting, if the substrate is flexible;

- $\quad$ simple and economical to manufacture using printed circuit production technology;

- do not disturb the aerodynamics of UAVs;

- $\quad$ easy integration in circuits;

- $\quad$ suitable for use in planar arrays.

The disadvantages compared to conventional antennas, are narrow bandwidth, losses in the conductor and dielectric, possible surface waves excitation, reducing the efficiency and limited power. Within the set of planar antennas, the present UAVs communication antennas designated by Switched Parasitic Array Antennas Radiator (SPAR) are interesting to achieve communication given the capacity to vary its radiation diagram.
During the last decade, smart antennas has been studied due to the advantages that they offer in the improvement of wireless communication [4]. These antennas are called SPAR and have the ability to control the radiation pattern and adjust the main lobe of radiation to a certain specified direction, and the null of radiation to the interfering signals. This process can be performed through reactance control using varicap diodes and is denoted by beamforming [6].

The ESPAR antenna uses mutual coupling to excite their parasitic elements. Each parasitic antenna element is associated with a variable reactance produced by two varicap diodes. The variation of the value of reactance is reflected in the variation of the maximum of antenna radiation, which can thus be directed to the receiver and direct the radiation nulls for unwanted signals as already indicated, which maximizes the signal to noise ratio (SNR) of the system [4][9].

\section{Planar Antennas}

Planar antennas began to be developed in the 1970s mainly for special applications such as radar, satellite communications, navigation systems, etc [10]. Currently, these have wide applicability in communication, because of their ease of construction, easy adaptation to various types of 
planar or non-planar surfaces and the possibility of suitable radiation patterns [10].

The need to develop antennas with little weight and size to be easily installed in the UAV wing as well as aerodynamic considerations, makes this type of antenna suitable to perform communication between UAV and ground station.

\subsection{Structure and basic characteristics of Planar \\ Antennas}

Conventional planar antennas generally consist of a metal patch or radiating element typically having a thickness $t<<\lambda_{0}$ (where $\lambda_{0}$ is the wavelength in free space) placed on a substrate of thickness $h$, usually with $0.003 \lambda_{0} \leq h \leq 0.05 \lambda_{0}$. The substrate is placed on a metallic plane, as shown in Fig (2). The radiating element and the mass-plane are made of a very thin layer of copper and the dielectric substrate that makes the separation of the metal plates generally has a dielectric constant $\left(\varepsilon_{r}\right)$ between $2.2 \leq \varepsilon_{r} \leq 12$, the value of (

\section{$\varepsilon_{r}$ ) depending on the [11] type of substrate.}

The planar antenna patch can have various geometries such as square, rectangular, circular, triangular, etc. The geometry and dimensions of the patch itself determines the antenna radiation characteristics [11] [12].

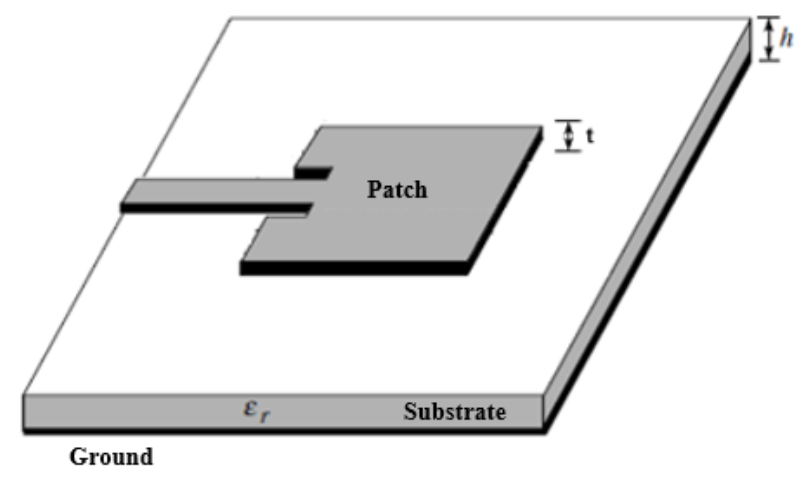

Fig. 2. Rectangular patch antenna geometry

\subsection{Method of transmission line}

The analysis of planar antennas is complex due to presence of non-homogeneous dielectrics, non-homogeneous boundary conditions, excitations diversity and numerous configurations of patch elements. Therefore, one resorts to models or analytical methods to enable a better understanding of the operating principles and characteristics of the antenna.

The full-wave methods generally are based on integral equations of the Sommerfeld type in the spectral domain and solutions of Maxwell's equations in the time domain. These methods have better accuracy when compared with analytical models, but its analysis is complex [11].

In the analytical method of the transmission line, the patch element is the resonant element of the transmission line where the characteristic impedance $\left(Z_{c}\right)$ and propagation phase constant $(\beta)$ are determined by the parameters of the antenna dimensions and substrate [11].

The effective dielectric constant $\left(\varepsilon_{\text {reff }}\right)$ varies with the change of the patch width $(W)$, however this depends on the operating frequency $\left(f_{r}\right)$, demonstrated in (8). At low frequencies it approaches the dielectric substrate constant value $\left(\varepsilon_{r}\right)$. The effective dielectric constant value can be calculated by the following equation (2). according to the following condition $W / h>1[11]$ :

$\varepsilon_{r e f f}=\frac{\varepsilon_{r}+1}{2}+\frac{\varepsilon_{r}-1}{2}\left[1+12 \frac{h}{W}\right]^{-\frac{1}{2}}$

Once the width $(W)$ and length $(L)$ patch are calculated, propagated electromagnetic fields along the radiating element present a discontinuity in the edges of the patch, and, diffracted fields (fringing fields) are generated at their ends, as shown in Fig. 3 a) and b). The amount of the fringing fields is a function of the dimensions of the patch, the thickness $(h)$ and substrate dielectric constant $\left(\varepsilon_{r}\right)[11]$.

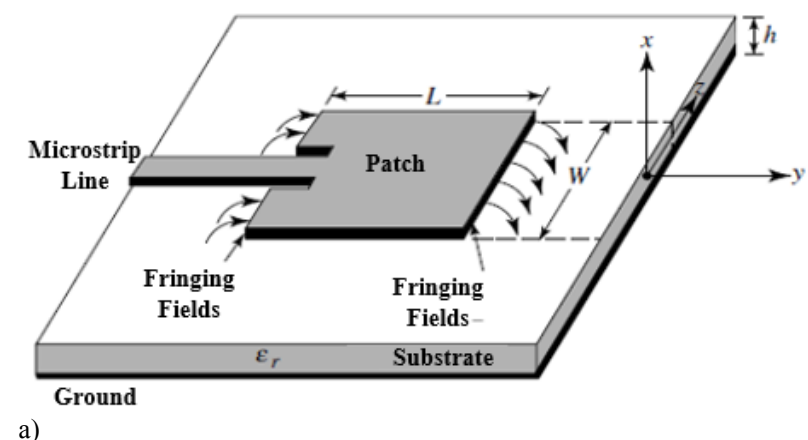

a)

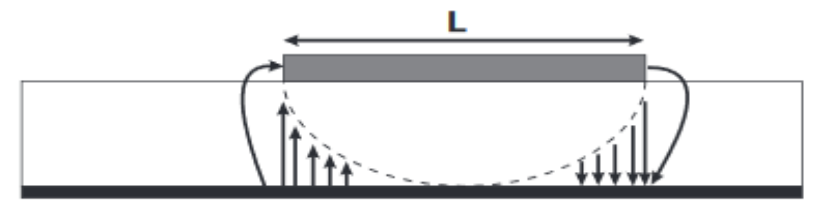

b)

Fig. 3. a) Planar antenna with rectangular geometry representation of fringing fields excited by a microstrip line b) Side view of electromagnetic fields propagated in substrate[11]

The behavior explained above, causes an electrical addition $\Delta L$ to the dimensions of the patch on both sides in the direction of the $x y$ plane (main plane E) as shown in figure (4).

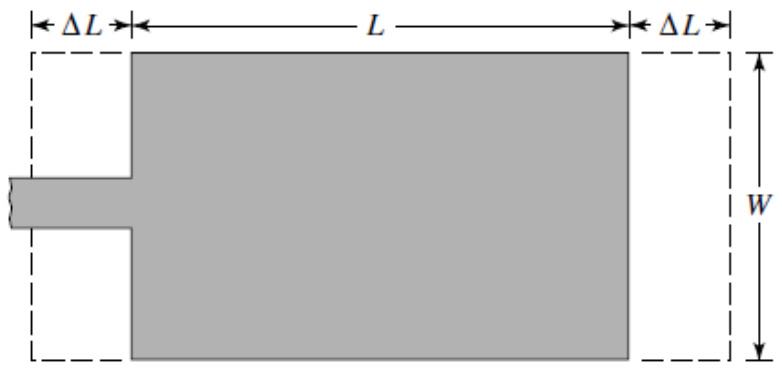

Fig. 4. Physical and affective length of the rectangular patch planar antenna[11]

This increase in the dimensions of the patch may be represented by two slots of width $(\Delta L)$ separated by a distance equal to the patch length. The length $(\Delta L)$ can be approximated according to [11] expression: 
$\frac{\Delta L}{h}=0.412 \frac{\left(\varepsilon_{\text {reff }}+0.3\right)\left(\frac{W}{h}+0.264\right)}{\left(\varepsilon_{\text {reff }}-0.258\right)\left(\frac{W}{h}+0.8\right)}$

Thus, the effective length of the patch is given by [11]:

$L_{e f f}=L+2 \Delta L$

For the dominant mode $T M_{010}$ the resonance frequency ( $f_{r}$ ) in the planar patch antenna is a function of length $(L)$ and is given by [11] the expression:

$$
\left(f_{r}\right)_{010}=\frac{1}{2 L \sqrt{\varepsilon_{r}} \sqrt{\mu_{0} \varepsilon_{0}}}=\frac{C_{0}}{2 L \sqrt{\varepsilon_{r}}}
$$

where $\left(C_{0}\right)$ is the speed of light in free space, $\left(\mu_{0}\right)$ the magnetic vacuum permeability whose value is $4 \pi * 10^{-7}$ and $\left(\varepsilon_{0}\right)$ the permittivity of vacuum with a value of $8.85418 * 10^{-12} C^{2} N^{-1} m^{-2}$. Equation (5) does not account for the edge effect. By taking this into account equation (6) should be modified to [11]:

$$
\left(f_{r c}\right)_{010}=\frac{1}{2 L_{e f f} \sqrt{\varepsilon_{r e f f}} \sqrt{\mu_{0} \varepsilon_{0}}}=q \frac{c_{0}}{2 L \sqrt{\varepsilon_{r}}}
$$

Being $(q)$ the edge effect factor, calculated as follows[11]:

$$
q=\frac{\left(f_{r c}\right)_{010}}{\left(f_{r}\right)_{010}}
$$

The patch width $(W)$ is related to the resonance frequency $\left(f_{r}\right)$ and dielectric constant $\left(\varepsilon_{r}\right)$ and it is calculated by the following equation[11]:

$$
W=\frac{1}{2 f_{r} \sqrt{\mu_{0} \varepsilon_{0}}} \sqrt{\frac{2}{\varepsilon_{r}+1}}=\frac{v_{0}}{2 f_{r}} \sqrt{\frac{2}{\varepsilon_{r}+1}}
$$

Neglecting edge effects the patch length is given by the following equation [11]:

$$
L=\frac{1}{2 f_{r} \sqrt{\varepsilon_{\text {reff }}} \sqrt{\mu_{0} \varepsilon_{0}}}-2 \Delta L
$$

The electrical addition ( $\Delta L$ ) originated by edge effects at the patch edges (slits), can be represented by an equivalent admittance $(Y)$ constituted by a conductance $(G)$ in parallel with a susceptance $(B)$ at each end of the patch[11] as shown in figure (5).

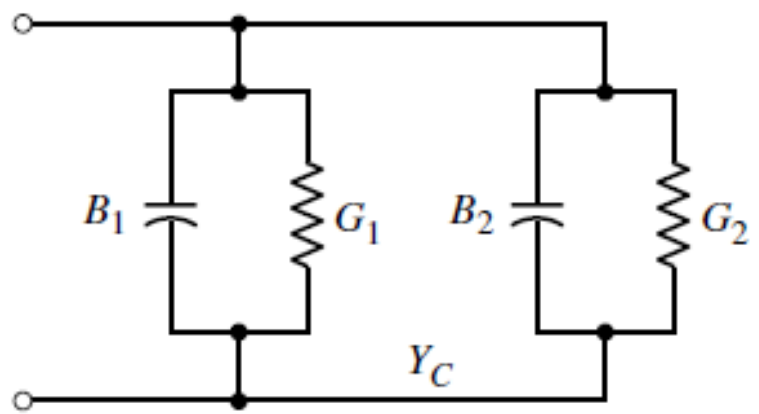

Fig. 5. Equivalent circuit of transmission line[11]

Considering patch slots numbered 1 and 2 as shown in figure (4.4), the equivalent admittance of the slot 1 is given by[11]

$Y_{1}=G_{1}+j B_{1}$

The values of the conductance ( $\left.G_{1}\right)$ and susceptance ( $B_{1}$ ) in slot 1 may be approximated by [11]:

$G_{1}=\frac{W}{120 \lambda_{0}}\left[1-\frac{1}{24}\left(k_{0} h\right)^{2}\right]$

$B_{1}=\frac{W}{120 \lambda_{0}}\left[1-0.636 \ln \left(k_{0} h\right)\right]$

Assuming the condition $\frac{h}{\lambda_{0}}<\frac{1}{10}$.

Considering the patch slots numbered 1 and 2 as shown in figure (4), in case the slot 2 is identical to slot 1 , the equivalent admittance is given by [11]:

$Y_{2}=Y_{1}, \quad G_{2}=G_{2}, \quad B_{2}=B_{1}$

The radiation power $\left(P_{r a d}\right)$ is given by [11]:

$P_{r a d}=\frac{\left|V_{0}\right|^{2}}{2 \pi \eta_{0}} \int_{0}^{\pi}\left[\frac{\sin \left(\frac{k_{0} W}{2} \cos \theta\right)}{\cos \theta}\right]^{2} \sin ^{3} \theta d \theta$

and $k_{0}=\frac{2 \pi}{\lambda}$ it is the propagation constant and $V_{0}=h E_{0}$.

The electric field for very thin thicknesses $\left(k_{0} h \ll 1\right)$ can be expressed by equation (15) [11]:

$E_{\theta} \simeq+j \frac{V_{0} e^{-j k_{0} r}}{\pi r}\left\{\sin \theta \frac{\sin \left(\frac{k_{0} W}{2} \cos \theta\right)}{\cos \theta}\right\}$ [11]:

The conductance may also be written from equation (16) 


$$
G_{1}=\frac{I_{1}}{120 \pi^{2}}
$$

where $I_{1}$ is given by[11]:

$$
I_{1}=\int_{0}^{\pi}\left[\frac{\sin \left(\frac{k_{0} W}{2} \cos \theta\right)}{\cos \theta}\right]^{2} \sin ^{3} \theta d \theta
$$

The input resonance admittance can be given by the following expression[11]:

$$
Y_{\text {in }}=2 G_{1}
$$

if the input admittance $\left(Y_{i n}\right)$ is real without the coupling effects, it can be expressed as [11]:

$$
Z_{\text {in }}=\frac{1}{Y_{\text {in }}}=R_{\text {in }}=\frac{1}{2 G_{1}}
$$

Considering the coupling effects, the input resonance resistance $\left(R_{i n}\right)$ is given by [11]:

$$
R_{i n}=\frac{1}{2\left(G_{1} \pm G_{12}\right)}
$$

where $G_{12}$ is the mutual conductance between the two slits, given by [11]:

$G_{12}=\frac{1}{120 \pi^{2}} \int_{0}^{\pi}\left[\frac{\sin \left(\frac{k_{0} W}{2} \cos \theta\right)}{\cos \theta}\right]_{0}^{2} J_{0}\left(k_{0} L \sin \theta\right) \sin ^{3} \theta d \theta$

$J_{0}$ is the $1^{\text {st }}$ Bessel's function of the $1^{\text {st }}$ kind and zero order.

The characteristic impedance $Z_{c}$ in the microstrip line can be given by the following expression[11]:

$$
Z_{c}=\left\{\begin{array}{cc}
\frac{60}{\sqrt{\varepsilon_{\text {reff }}} \ln \left[\frac{8 h}{W_{0}}+\frac{W_{0}}{4 h}\right],} & \frac{W_{0}}{h} \leq 1 \\
\frac{120 \pi}{\sqrt{\varepsilon_{\text {reff }}}\left[\frac{W_{0}}{h}+1.393+0.667 \ln \left(\frac{W_{0}}{h}+1.444\right)\right]}, & \frac{W_{0}}{h}>1
\end{array}\right.
$$

considering $Z_{c}=50 \Omega$, the width of the microstrip line $\left(W_{0}\right)$ can be calculated by solving the equations (22) or (23) in order to $W_{0}$.

The input resonance resistance for a point $\left(y_{0}\right)$ can be approximated by the expression [11]:

$$
R_{i n}\left(y=y_{0}\right)=\frac{1}{2\left(G_{1} \pm G_{12}\right)} \cos ^{2}\left(\frac{\pi}{L} y_{0}\right) \Leftrightarrow R_{i n}(y=0) \cos ^{2}\left(\frac{\pi}{L} y_{0}\right)(24)
$$

Solving this last expression in order to $\left(y_{0}\right)$ it is possible to determine the location of the inset feed point, which corresponds to the distance from the slot patch 1 to the inset feed point in order to adapt the impedance of the antenna ( $\left.Z_{c}=50 \Omega\right)[11]$. In (6) the inset feed technique for impedance matching is shown.

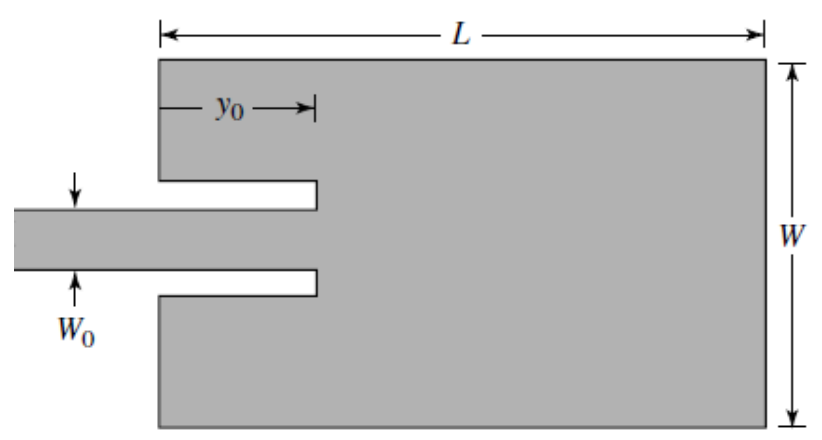

Fig. 6. Planar antenna rectangular geometry adapted to the inset feed technique[11]

\subsection{Excitation Methods}

The settings that can be used to excite the antenna can be classified into two groups. A direct contact group and another one by an energy coupling or indirect [8]. The direct way is usually by microstrip line or coaxial cable, also known as probe method and the indirect one by aperture coupling or proximity coupling [8] [13].

The choice of excitation method is a determining factor for the adjustment of the antenna. [8] Each of these methods is shown in the figures (7) to (9).

The excitation through the microstrip line is made through a conductive line with a width much smaller than the patch as shown in figure ( 3 a). The conductive line is printed on the same antenna substrate plane. This form of excitation has the following properties [8] [11]: it is simple to construct; easy input impedance adaptation by the inset feed technique; as the microstrip line is on the same structure of the patch it causes discontinuities in the microstrip line that gives rise to decreasing spurious radiation and efficiency of the antenna. It also has, when compared to other excitation methods including coupling, a lower bandwidth.

The probe excitation consist in an internal connector (coaxial) connected to the patch through the substrate to the ground plane (external connector), usually by a soldering point. [11]. This method is represented in Figure (7).

Its main features are [11] [12] the power supply is isolated from the rest of the antenna decreasing the spurious radiation, which results in greater efficiency; for substrates with higher thickness it becomes more difficult to apply; has a smaller bandwidth compared to other methods; the probe method also allows a simple and similar way as the previous method to perform the antenna impedance matching, by placing the antenna excitation point at any location of the patch.

This type of excitation uses two layers of substrate, placing the patch on top of the structure and the microstrip line positioned between the two dielectric [12]. Usually the lower substrate possesses a higher dielectric constant $\left(\varepsilon_{r}\right)$ than the substrate placed on top. The antennas fed by this method have a greater bandwidth than the antennas fed by the aforementioned methods given the increased thickness of 
the substrate. However, the power supply is not fully isolated, there is still spurious radiatio

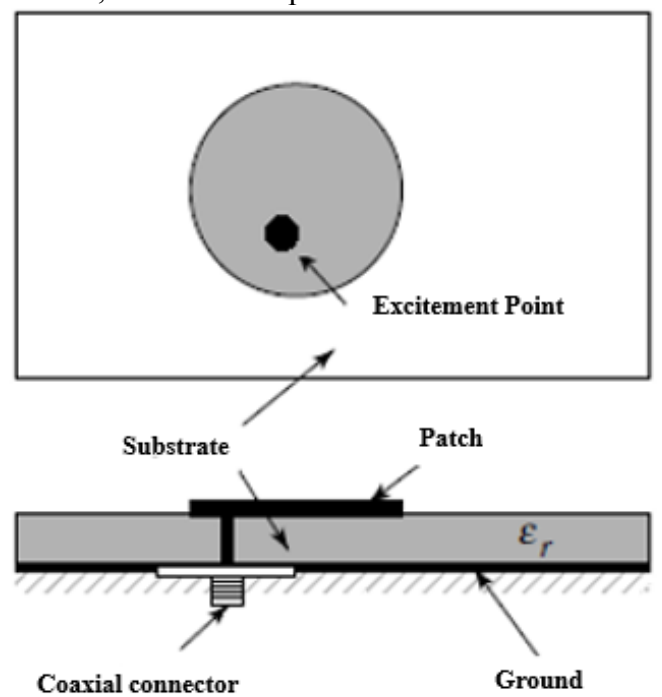

Fig. 7. Planar antenna fed by probe.

$\mathrm{n}$, but to a lesser extent than with the previous methods.

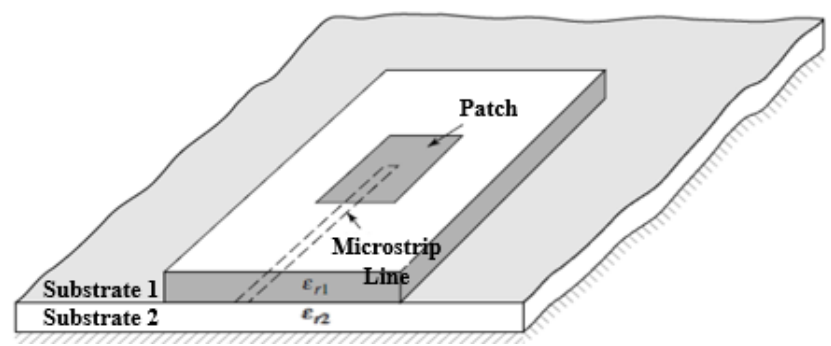

Fig. 8. Planar antenna fed by Aperture-coupled

The aperture coupling technique is similar to proximity coupling technique, it consists of two separated substrates through a ground plane. At the bottom of the substrate positioned below, there is a microstrip line whose energy is coupled to the patch through a slot in the ground plane separating the two substrates, this slot is usually centered below the patch. The bottom substrate usually has a dielectric constant $\left(\varepsilon_{r}\right)$ higher than upper substrate in order to reduce unwanted radiation. The ground plane between substrates isolates the power to the patch minimizing interference from spurious radiation [11]. In (9) this kind of excitation method is represented:

\section{Proposal Planar Antenna ESPAR}

After the theoretical explanation of planar antennas and defined the structure and method of excitation, becomes necessary to conduct the study and simulation of the proposed antenna. This section aims to study, simulate and develop the planar antenna ESPAR called P-ESPAR.

Operational requisites of planar ESPAR antenna that must meet the descending UAV communication conditions: frequency of resonance $(1.33 \mathrm{GHz})$; band width greater than or equal $(8.7 \mathrm{MHz})$ and stationary wave ratio $(<-10 \mathrm{~dB})$.

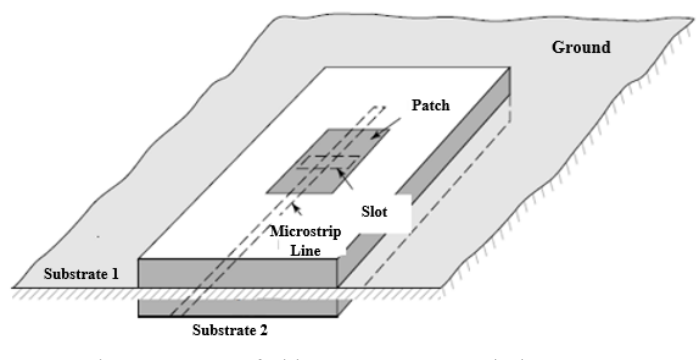

Fig. 9. Planar antenna fed by Aperture-coupled

4.1. The planar antenna element configuration (patch) The configuration of the planar antenna began with the analysis of various dielectric substrates in order to choose the most suitable for the project.

The substrate chosen for the project was the RT Duroid (5870), with $\left(\varepsilon_{r}=2.33 \pm 0.02, \tan \delta=0.0005 a 0.0012\right.$ and a thickness $h=1.575 \mathrm{~mm})$.

Subsequently the antenna is dimensioned theoretically with the above mathematical expressions using the transmission line method. This phase is important to analyze the antenna behavior with the change of its dimensions. The antenna will be simulated and optimized by CST Microwave Studio program. The CST MWS program is an electromagnetic simulator based on full wave methods (EM) with high performance. It integrates simulation, visualization, modeling and automation in an easy environment used to solve 3D EM problems accurately.

After the theoretical dimensions were calculated, the planar antenna was drawn using the MWS CST program and was an optimization tool designated by Optimazer Transient parameter, which makes multiple scans of the parameters that constitute the dimensions of the patch in order to improve the fit and performance o the antenna, was used to obtain a low value of the standing wave ratiomodule for 1.33 $\mathrm{GHz}$.

Within the optimized parameters it was found that the most important parameter for the antenna behavior is the patch length $(L)$, as can be observed in figure (10).

As can be seen, the variation of the parameter $L$ drastically changes the behavior of the antenna. Note that for higher values of $L$, the resonance frequency decreases considerably. This is in accordance with equation (9) where $L$ is inversely proportional to the resonance frequency.

In figure (11) is shown the drawing in CST MWS, of the optimized planar antenna with only one element:

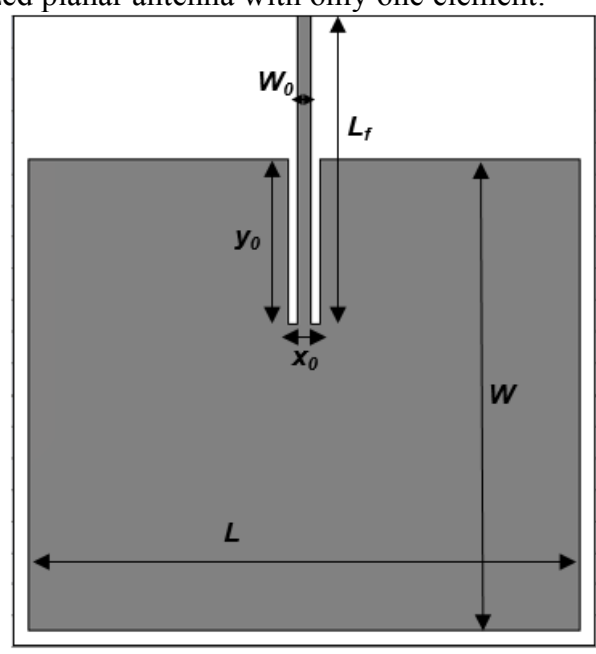

Fig. 11. Representation of antenna in CST MWS with $(\mathrm{W}=87.4$. $\mathrm{L}=74, \mathrm{~W} 0=2.3, \mathrm{y} 0=26.1, \mathrm{x} 0=4, \mathrm{Lf}=60$ ) all dimensions in $\mathrm{mm}$. 
Pedro Marques, Maria Martins, António Baptista and João Paulo N. Torres/

Journal of Engineering Science and Technology Review 11 (1) (2018) 90-102

In figure (12) it is found that the antenna is fully adapted

to the desired frequency band $f_{r}=1.334 \mathrm{GHz}$. As mentioned above, the adaptation level is represented by the stationary wave ratio module, $\left|S_{11}\right|_{d B}$.

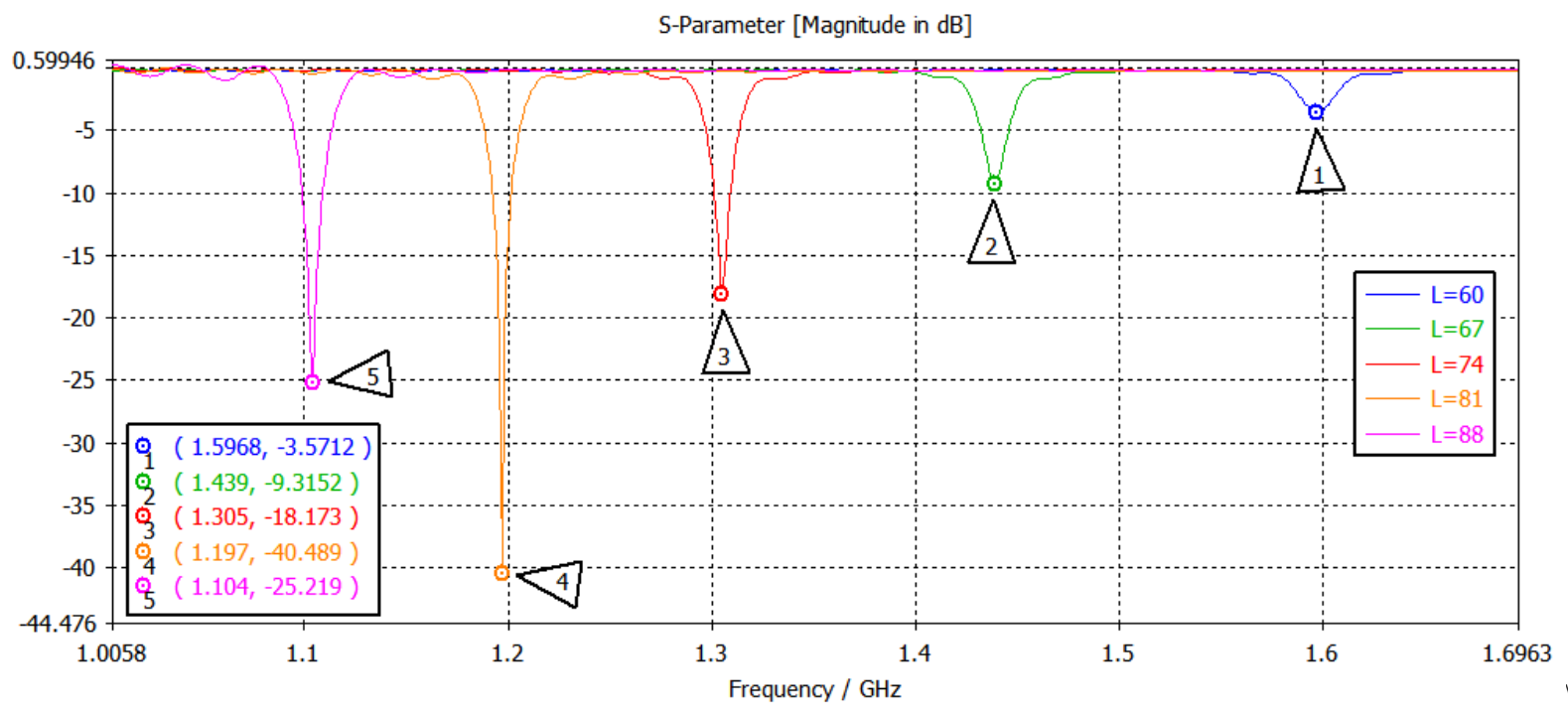

Fig. 10. Graph of change of $\left|S_{11}\right|_{d B}$ a function of frequency for different values of $L$ versus frequency

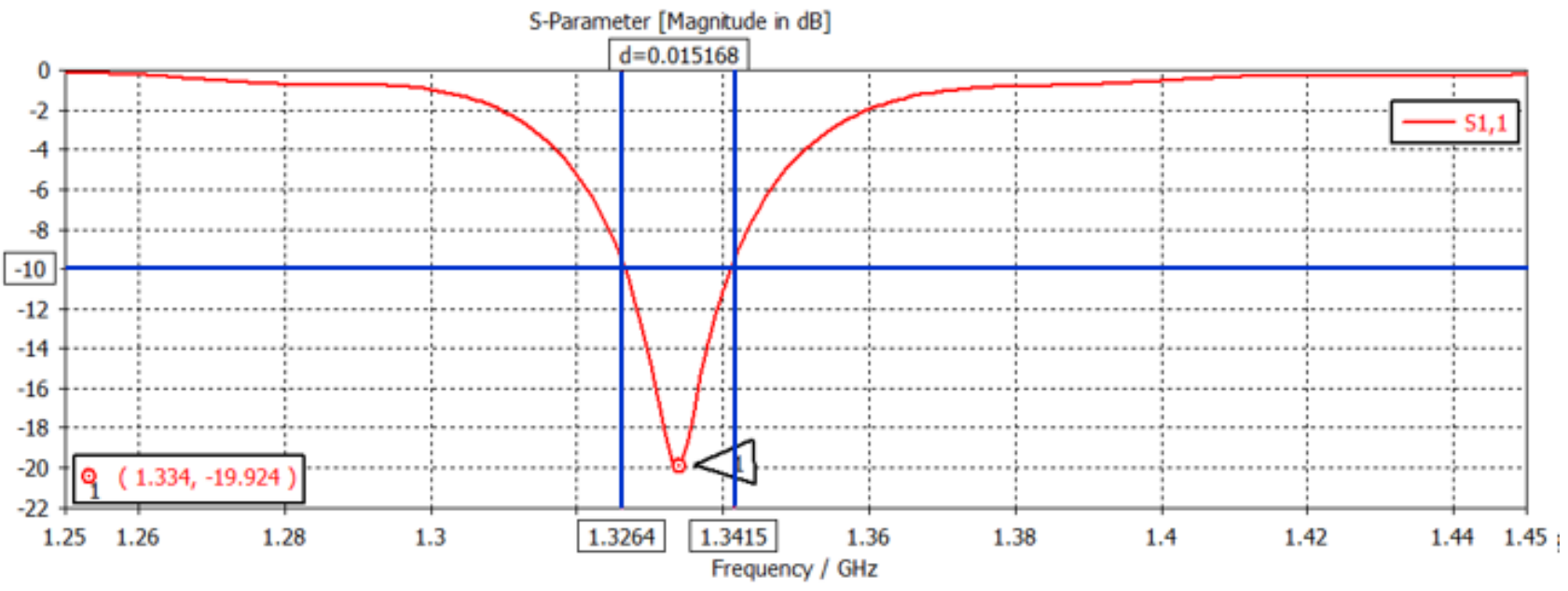

Fig. 12. Graphical representation of $\left|S_{11}\right|_{d B}$ a function of frequency for dimensions of the planar antenna single-patch optimized

For this case it was obtained $\left|S_{11}\right|=-19.924 d B$ satisfying the criterion proposed initially $\left|S_{11}\right| \leq-10 d B$. The planar antenna with a single patch has a bandwidth of ( $\mathrm{MHz} 15.14)$, which complies with requisites.

In fig. 13 we can see the radiation characteristics in the plane $\mathrm{E}(\mathrm{Phi}=0)$ and $\mathrm{H}($ theta $=90)$. The antenna has a gain of $5.44 \mathrm{~dB}$ a beam width at half power bandwidth (HPBW) $(-3 \mathrm{~dB})$ of $95.4^{\circ}$, in plane $\mathrm{E}$ and in the $\mathrm{H}$ plane is about $126.1^{\circ}$.

\section{P-ESPAR Antenna}

Two or more patches may constitute an antenna array in order to improve certain desired characteristics, such as bandwidth, directivity and gain. These characteristics cannot be improved with only a single element. In [8] it is mentioned that the antenna array's radiated power can be concentrated in a smaller area, which translates into an increase of antenna directivity. Besides this parameter is the gain and half power beam width are also improved.

However, the array of antennas alone is not able to perform the specifics of the originally proposed antenna, this means that a reconfigurable antenna capable of changing the direction of the main beam.

The antenna P-ESPAR consists of a cluster of three patch elements, the active element corresponds to the central patch, excited by a microstrip line similar to the previous antenna with only one element. The two other patches, designated as parasitic patches, have dimensions identical to the active element and are disposed on each side of the central element. The mutual coupling between the patches is controlled using four varicap diodes.

The varicap diodes introduce capacitive reactances that vary, as the reverse bias voltage is applied to the terminals of the diodes. This phenomenon allows for the adjustment of 
mutual coupling between the patches, which results in a change of the effective length $(L)$ of the antenna, or the effective length of the two parasites patches. This change is manifested in the variation of the amplitude and current phase of the parasitic elements in relation to the active element which in turn varies the slope of the main beam [13]. The diodes varicap $B B 833$ were chosen for simulation purposes.
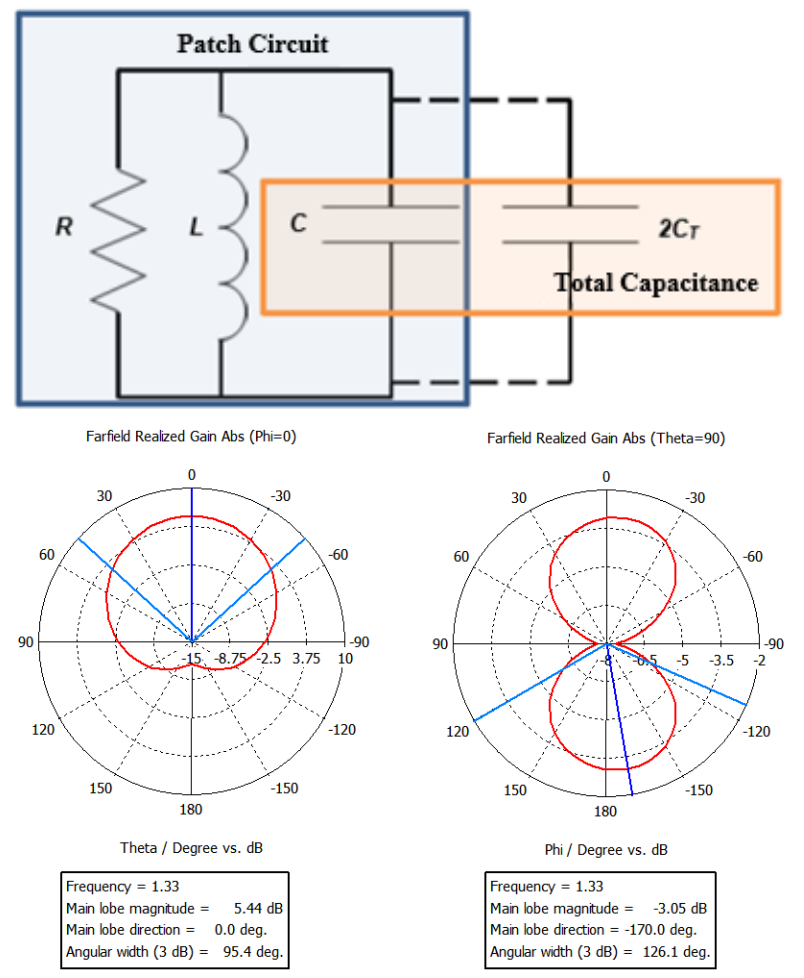

Fig 13. Polar representation of the radiation pattern of the antenna single-patch optimized in the plane $\mathrm{E}$ and $\mathrm{H}$

Therefore, analyzing the behavior of the antenna with the addition of varicap diodes is essential to understand how the parameters of the antenna vary. In this sense it is presented a simple equivalent circuit model that helps to analyze this behavior.

The equivalent circuit model of the planar antenna without diodes may be represented by an RLC equivalent circuit in parallel, as illustrated in Fig. 14 [14]. To determine the values of $L$ and $C$, full wave simulation of electromagnetic fields by the CST MWS program was used. $L$ and $C$ represent the magnetic and electric energy stored in the resonant circuit. The capacity of the varicap diode is represented as a combination of capabilities in parallel of the two diodes positioned between the patches $\left(2 C_{T}\right)$ as shown in Fig. 14.

The resonant frequency $\left(f_{0}\right)$ without the diode, that is, the unloaded circuit is given by equation (25) and occurs at resonance [14]:

$$
2 \pi f_{0}=\frac{1}{\sqrt{L C}}
$$

As a result of loading the capacity of the varicap diodes, the circuit capacity becomes the combination of $\mathrm{C}$ parallel to $2 C_{T}$.

In this case the resonance frequency $\left(f_{c}\right)$ of the circuit is given by [14]:

$$
2 \pi f_{c}=\frac{1}{\sqrt{L\left(C+2 C_{T}\right)}}
$$

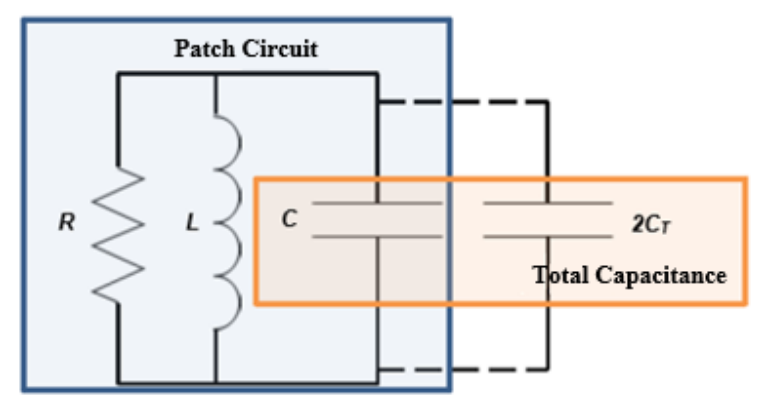

Fig. 14. Equivalent circuit of planar antenna with varicap diodes

Since the value of $C_{T}$ is known and $f_{c}$ can easily be determined by a simulation of $\left|S_{11}\right|$ using CST MWS the value of $\mathrm{C}$ can be calculated by [14]:

$$
C=\frac{2 C_{T}}{\left(f_{0} / f_{c}\right)^{2}-1}
$$

The P-ESPAR antenna configuration takes into account various critical parameters as the choice of substrate, its dimensions, the excitation method, the selection of the varicap diodes, the capacitive reactance adjustment necessary for the required specifications, and the position of coupling of the diodes. This design process is depicted in Fig. 15.

Fig. 15. a) Process study and development of P-ESPAR antenna

The final structure of the antenna consists of an array of three identical patches positioned side by side, the space between the patches corresponding to the length of the varicap diode $\mathrm{G}=2.5 \mathrm{~mm}$. The central patch is the active element and the side to this parasitic element. The active element is excited by Wave port tool of CST Microwave studio program already used in the antenna with a single element. The antenna substrate is RT Duroid 5780 having a thickness of $h=1.575 \mathrm{~mm}$.

The process of determining the dimensions of the PESPAR antenna elements was conducted similarly to the one for the sigle patch, using optimization tool of CST MWS program designated Optimazer Transient parameter.

As the mutual coupling position $(\mathrm{O})$ is essential in balancing all the parameters of the antenna, mainly the resonance frequency, bandwidth and adaptation, several simulations were performed particularly of $\left|S_{11}\right|_{d B}$. It was verified that for smaller proximity of the varicap diodes the level of variation of the main parameters was smaller and 
smaller as the capacitive reactance's of the diodes were maintaining the operational requirements of the antenna. changed as shown in Fig. 16. This factor is important for

S-Parameter [Magnitude in $\mathrm{dB}$ ]

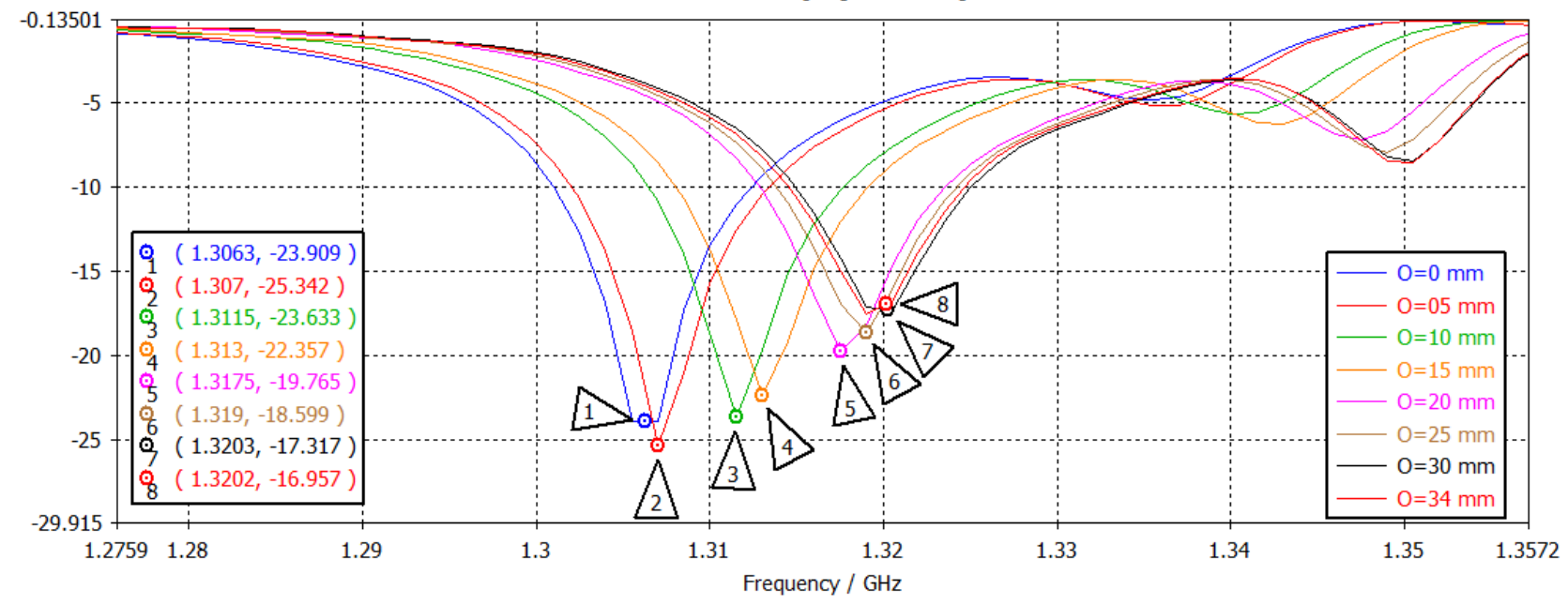

Fig. 16. Graphical representation of $\left|S_{11}\right|_{d B}$ variation for different values O.

After several simulations it was found that the best equilibrium was achieved with $\mathrm{O}=31 \mathrm{~mm}$.

Although the Fig. 16 takes into account only the capacity $C=1 p F$ note that the resonance frequency approaches the desired $1.33 \mathrm{GHz}$ with increasing values. Only after choosing the coupling position, the antenna optimization P-ESPAR, was completed.

Figure (17 $\mathrm{a}$ and $\mathrm{b})$ represent the final design of the proposed antenna seen from above and sideways, respectively. The images correspond to the design made in the CST MWS program.
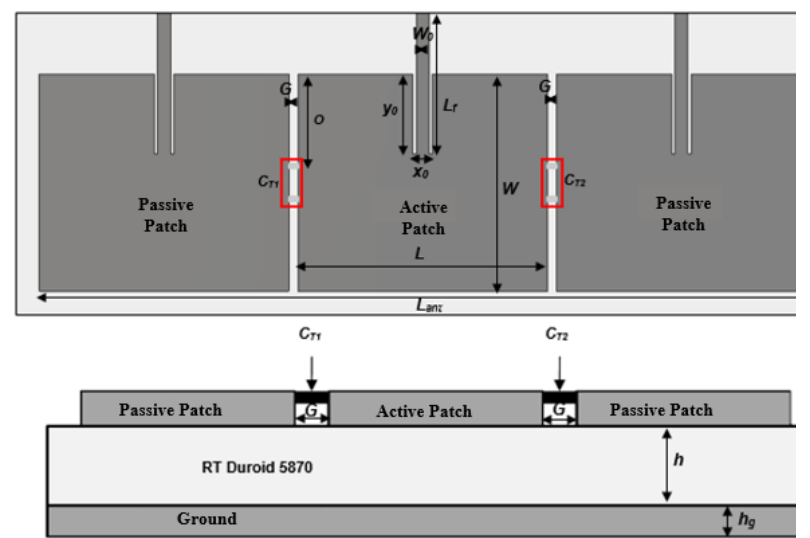

Fig.17. a) Structure and dimensions of P-ESPAR antenna in perspective view from above b) P-ESPAR antenna on the side perspective

\section{Simulation P-ESPAR Antenna}

The antenna design shown in figure (17 a and b) was simulated using CST MWS program for the frequency of $1.33 \mathrm{GHz}$. Following the process shown above in figure (16).

The values of the capacities of the varicap diodes were set after numerous simulations in order to find the ideal characteristics that fulfill the operational requirements for each situation of configuration of the radiation beam. The azimuths chose were: $-25^{\circ},-20^{\circ},-15^{\circ},-10^{\circ},-5^{\circ}, 5^{\circ}, 10^{\circ}, 15^{\circ}$, $20^{\circ}$ and $25^{\circ}$ in relation to its reference azimuth $\left(0^{\circ}\right)$. The variable capacity $\left(\mathrm{C}_{\mathrm{T} 1}\right)$ corresponding to all the junction capacities of the two varicap diodes in the left position of the central patch while $\left(\mathrm{C}_{\mathrm{T} 2}\right)$ corresponds to the set of the junction capacities of diodes positioned on the right side of the central patch, as illustrated in figure $(17 \mathrm{a})$. To an identical mutual coupling in each of the parasitic patches it has always been considered the same value in the two variable capacity diodes which constitute $\left(\mathrm{C}_{\mathrm{T} 1}\right)$ or $\left(\mathrm{C}_{\mathrm{T} 2}\right)$ to avoid asymmetries in the mutual coupling. The nine simulated radiation settings are shown in table (1).

Table 1. Antenna characteristics for each P-ESPAR radiation setting

\begin{tabular}{|c|c|c|c|c|c|c|}
\hline \multirow[t]{2}{*}{ Main Lobe [] } & \multicolumn{6}{|c|}{ P-ESPAR Antenna Characteristics } \\
\hline & Gain[dBi] & $\mathbf{f}_{\mathrm{c}}[\mathbf{G H z}]$ & $\left|\mathbf{S}_{11}\right|[\mathrm{dB}]$ & LB[MHz] & $\mathrm{C}_{\mathrm{T} 1}[\mathrm{pF}]$ & $\mathrm{C}_{\mathrm{T} 2}[\mathrm{pF}]$ \\
\hline-25 & 5.42 & 1.31 & -27.285 & 10 & 1.7 & 2.4 \\
\hline-20 & 5.36 & 1.307 & -23.42 & 9.65 & 1.5 & 2.6 \\
\hline-15 & 6.79 & 1.301 & -14.821 & 9.23 & 1.1 & 2.8 \\
\hline-10 & 4.09 & 1.301 & -20.028 & 9.65 & 2.1 & 2.7 \\
\hline-5 & 4.97 & 1.315 & -23.164 & 11.4 & 1.3 & 2.1 \\
\hline 0 & 5.42 & 1.298 & -18.771 & 9.2 & 2.6 & 2.6 \\
\hline 5 & 4.96 & 1.315 & -22.738 & 11.4 & 2.1 & 1.3 \\
\hline 10 & 4.01 & 1.301 & -19.983 & 9.65 & 2.7 & 2.1 \\
\hline 15 & 6.72 & 1.301 & -14.896 & 9.23 & 2.8 & 1.1 \\
\hline 20 & 5.28 & 1.305 & -23.383 & 10.1 & 2.6 & 1.5 \\
\hline 25 & 3.62 & 1.31 & -26.284 & 10 & 2.4 & 1.7 \\
\hline
\end{tabular}


Pedro Marques, Maria Martins, António Baptista and João Paulo N. Torres/

Journal of Engineering Science and Technology Review 11 (1) (2018) 90-102

Analyzing the table it appears that there is symmetry in the values of the varicaps for each radiation beam configuration (beamforming). For example, for $-25^{\circ}$ direction of the main lobe is $\mathrm{C}_{\mathrm{T} 1}=1.7 \mathrm{pF}$ and $\mathrm{C}_{\mathrm{T} 2}=2.4 \mathrm{pF}$, whereas to $25^{\circ}$ the values of the variable capacitors are switched. This phenomenon occurs due to the existence of symmetry in the patches dimensions.

In figures 18 and 19 simulations are presented simultaneously to $\left|S_{11}\right|_{d B}$ versus frequency and the radiation patterns corresponding to the upper and lower direction of the main lobe of the antenna.

Fig. 19 shows that there are great fluctuations in the resonance frequency, for $0^{\circ}$ corresponds to $f_{c}=1.298 \mathrm{GHz}$, while that for the maximum and minimum values achieved in the variation of the main lobe $\left(-25^{\circ}\right.$ and $\left.25^{\circ}\right) f_{c}=1.31 \mathrm{GHz}$, existing consistency of operational requirements.
Farfield Realized Gain Abs (Phi $=0$ )

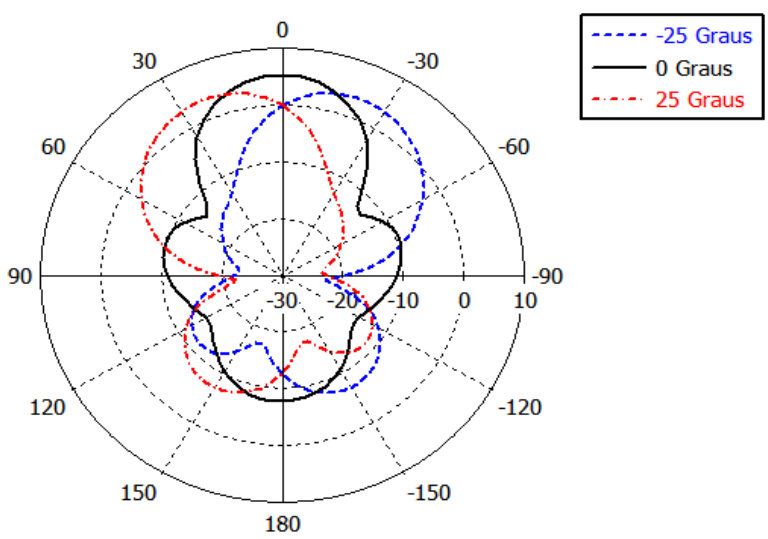

Theta / Degree vs. dB

Fig. 18 - Graphical representation radiation pattern in the plane and to $25^{\circ}, 0$ and $25^{\circ}$.

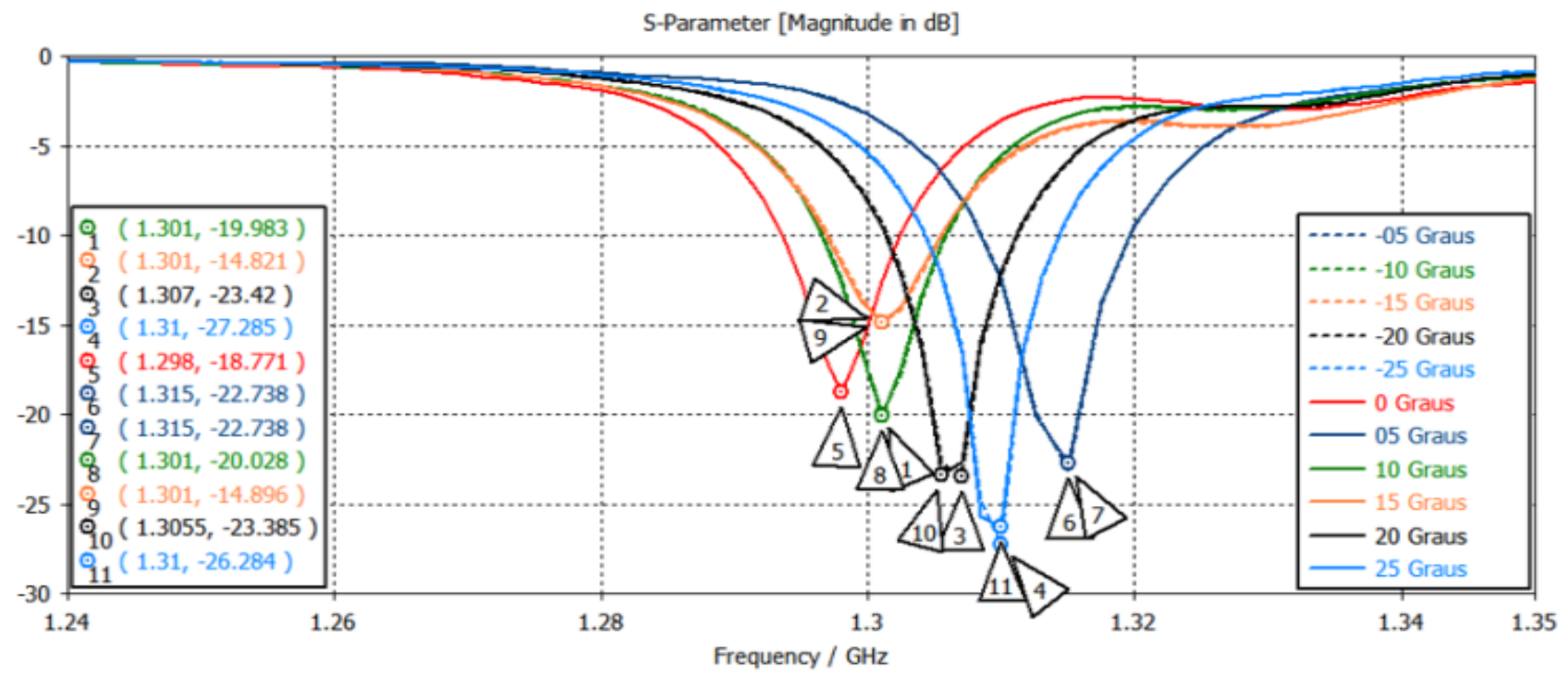

Fig. 19. Graphical representation of $\left|S_{11}\right|_{d B}$ a function of frequency for various configurations radiation

Now, all the simulations of $\left|S_{11}\right|_{d B}$ and radiation diagrams corresponding to the radiation beam settings achieved by the P-ESPAR antenna will be presented.

Looking at the charts $\left|S_{11}\right|_{d B}$ of figures 18 and 19 it is found that the resonance frequency remains virtually unchanged and close to the value of $1.33 \mathrm{GHz}$. The bandwidth of all the antenna radiation patterns of varying configurations of the P-ESPAR antenna meets the $8.7 \mathrm{MHz}$.

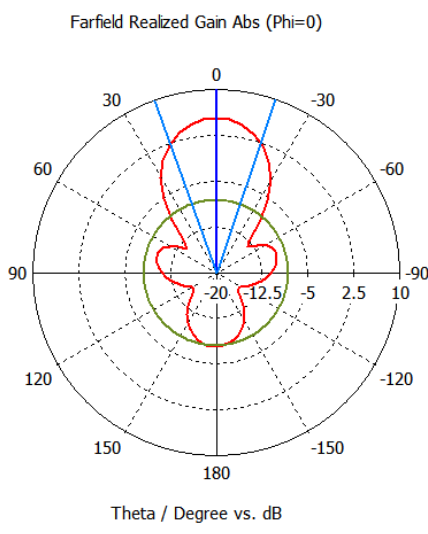

Frequency $=1.33$ Main lobe magnitude $=5.42 \mathrm{~dB}$ Main lobe direction $=0.0 \mathrm{deg}$. Side lobe level $=-13.5 \mathrm{~dB}$
In Fig. 19 shows the graph of $\left|S_{11}\right|_{d B}$ as a function of frequency for the multiple radiation beam configurations. Observing the graph of figure (18) it is found that the curve which describes a given beam direction is identical to the curve that describes the symmetrical direction, this is, there is symmetry in mutual coupling. This is due to the symmetry of the patches, cited above. Next, are presented the radiation patterns for each configuration of the radiation of P-ESPAR antenna. 
Pedro Marques, Maria Martins, António Baptista and João Paulo N. Torres/

Journal of Engineering Science and Technology Review 11 (1) (2018) 90-102

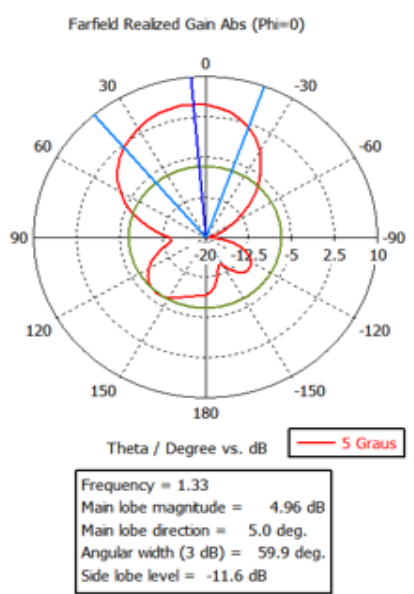

b)
Farfed Reaked Gan Abs (Phi=0)

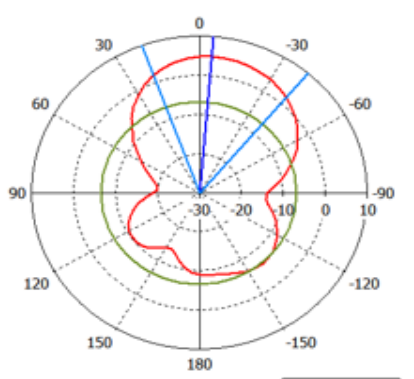

Theta / Degree vs. dB -5 Graus

Frequency $=1.33$

Man bobe magntude - $4.97 \mathrm{~dB}$ Main lobe drection $=-5.0$ deg. Angular wiơth $(3 \mathrm{~dB})=60.1 \mathrm{deg}$. Side lobe level $=-11.6 \mathrm{~dB}$

c)

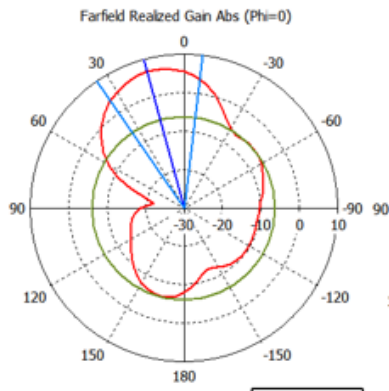

Theta / Degree vs. dB -15 Grous

Frequency $=1.33$

Main lobe magntude $=6.72 \mathrm{~d}$ Man lobe drection $=15.0$ deg. Angular width $(3 \mathrm{~dB})=41.4 \mathrm{deg}$ side bobe level $=-12.9 \mathrm{~d}$ a)

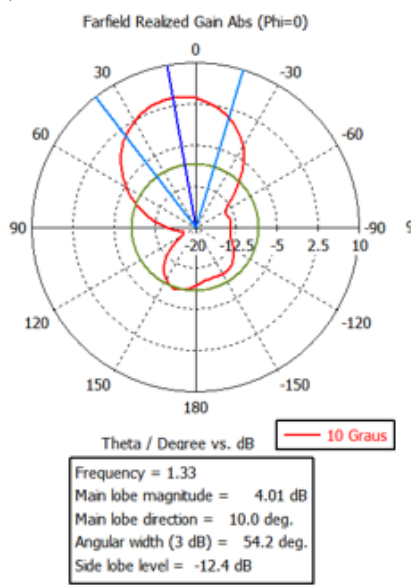

d)

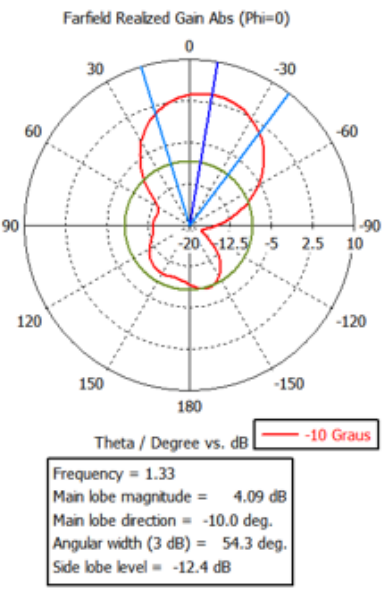

e)

f)

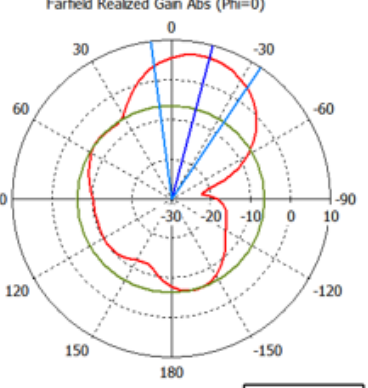

Theta / Degree vs, dB - 15 Graus

Frequency $=1.33$

Man lobe magntude $=6.79 \mathrm{~dB}$ Main lobe drection $=-15.0$ deg. Angular woth $(3 \mathrm{~dB})=41.5 \mathrm{~d}$
side bbe level $=-13.1 \mathrm{~dB}$

g)

Fig. 20. Radiation pattern in the plane E for: a) $0^{\circ}$ b) $5^{\circ}$ and c) -5 ) d) $10^{\circ}$ e) $-10^{\circ}$ f) $15^{\circ}$ g) -15 .

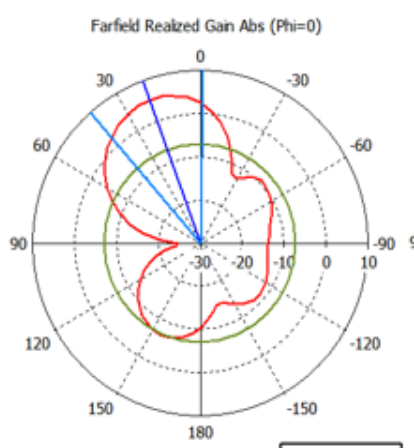

Theta / Degree vs. dB -20 Grous

Frequency $=1.33$

Man lobe magntude $=5.28 \mathrm{de}$ Man lobe drection $=20.0 \mathrm{deg}$. Angular with $(3 \mathrm{~dB})=41.8$ deg. Side lobe level $=-12.3 \mathrm{~dB}$

a)
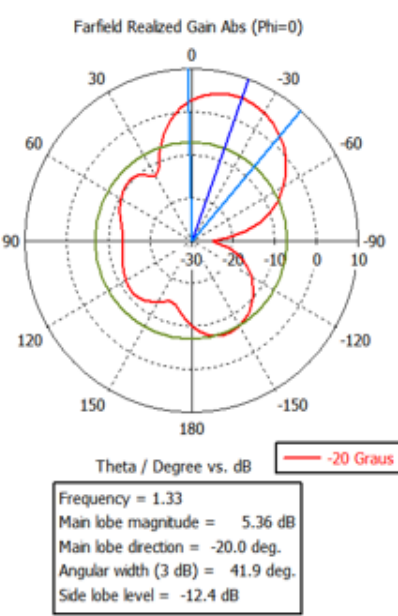

b)

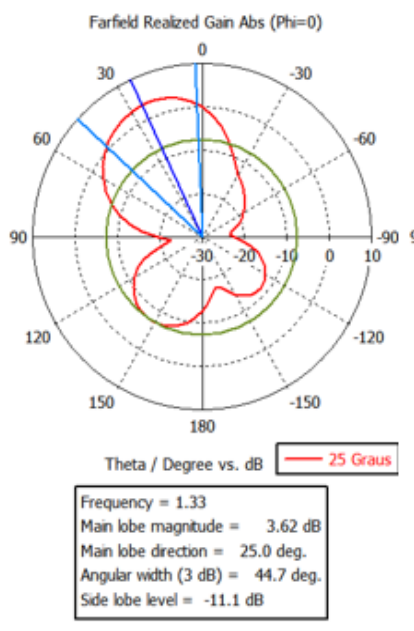

c)

Fig. 21. Graphical representation radiation pattern in the plane $E$ to a) $20^{\circ}$ b) $-20^{\circ}$ c) $25^{\circ}$ d) $-25^{\circ}$

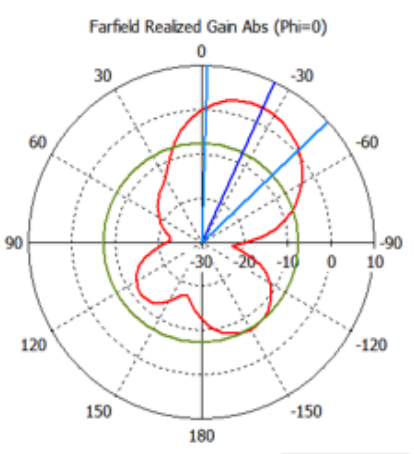

Theta / Degree vs. dB -25 Graus

Frequency $=1.33$

Man lobe magntude $=3.7 \mathrm{~dB}$ Main lobe drection $=-25.0 \mathrm{deg}$. Angular width $(3 \mathrm{~dB})=44.7 \mathrm{deg}$. side lobe level $=-11.1 \mathrm{~dB}$

d)
Through the radiation patterns represented by figures 20 and 21 it can be verified that the characteristic variation of the direction of the main lobe of the P-ESPAR antenna was reached. In the direction $15^{\circ}$ and $-15^{\circ}$ corresponding to a maximum gain of $6.72 \mathrm{~dB}$ to $6.79 \mathrm{~dB}$, respectively, whereas, in the direction $25^{\circ}-25^{\circ}$ its gain corresponds to only $3.62 \mathrm{~dB}$ and $3.7 \mathrm{~dB}$. The level of secondary lobes lies on an average value of $-12 \mathrm{~dB}$. It is to be noted that in practice the PESPAR antenna nulls will not emit radiation in the rear direction due to the placement of the antenna on the aircraft wing.

\section{Simulation P-ESPAR Antenna}

This section will present the P-ESPAR antenna design, which comprises the front, side and rear view of the antenna with the respective dimensions. The project was carried out through the 3D CAD design program called Solidworks. The points that define the antenna in the CST MWS program were imported into the Solidworks program facilitating the design of the antenna. The dimensions are presented on a millimeter scale. In addition to the P-ESPAR antenna, an UAV wing profile was also designed in Solidworks. 
In Fig. 22 it is shown the layout front of the P-ESPAR antenna. As shown in the figure 21 there are 8 holes of $1 \mathrm{~mm}$ diameter. These holes indicate the passage of conductors needed for inversely polarizing the diode varicap. The diodes are placed between the holes in order to prevent any future interference between the wires, that is, considering a diode varicap the bottom hole to this, connects to its cathode, while the upper hole connects to the anode.

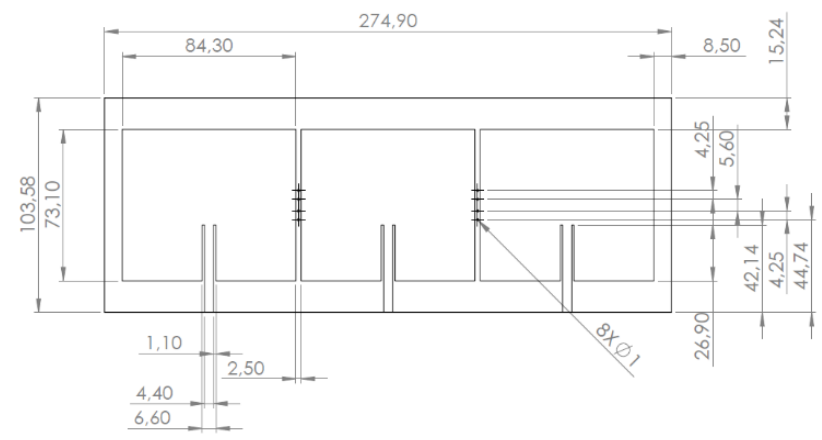

Fig. 22. Representation of the front configuration of P-ESPAR antenna

In Fig. 23 the layout side of the P-ESPAR antenna as shown. Due to the scale in the Fig. 23 the ground plane and the patches cannot be seen, therefore detailed views A and B on a scale of 50:1 are provided. The detail $\mathrm{A}$ is the scale expansion at point $\mathrm{A}$, noting that item 3 indicates the patch and item 2 is the dielectric substrate. While the detail $\mathrm{B}$ is the scale expansion of point $B$, where item 2 indicates the dielectric substrate as in the detail and the item 1 the ground plane.

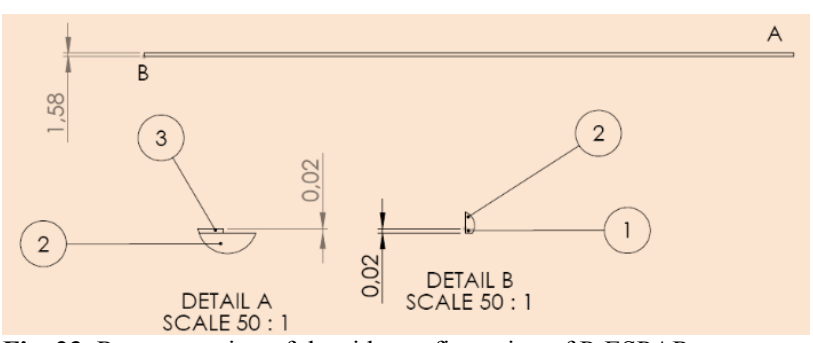

Fig. 23. Representation of the side configuration of P-ESPAR antenna with its details A and B.

In Fig. 24 it is represent the rear layout of the P-ESPAR antenna constituted by holes. It must be noted that the holes contain a circle centered at each central point of the holes' diameter of $3 \mathrm{~mm}$. In this place around the holes there is no ground plane in order to avoid interference from wires with the ground plane.

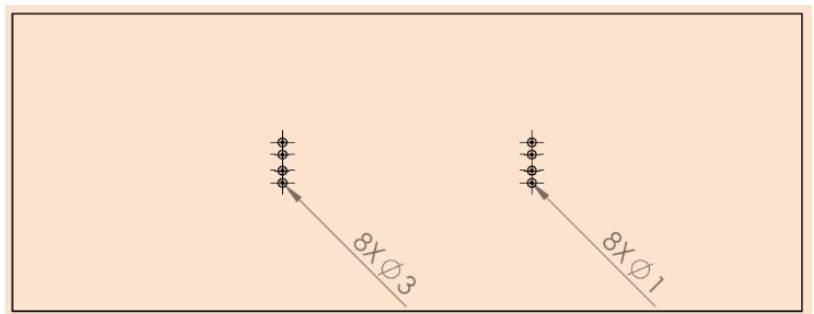

Fig. 24. Representation of rear configuration of P-ESPAR antenna.

In Fig. 25 an image of the UAV wing with the P-ESPAR antenna is presented, this figure was made using the SolidWorks software.

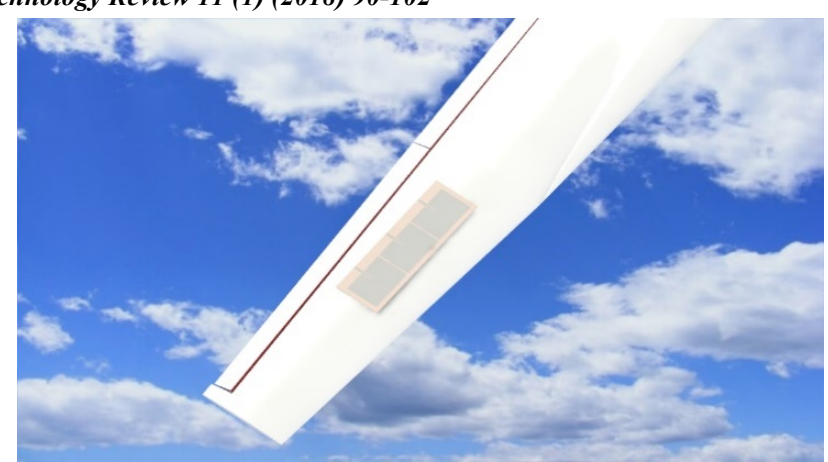

Fig. 25. Photography performed by SolidWorks program in a full scale representing the wing UAV with P-ESPAR antenna placed.

\section{Conclusions}

Militarily, the use of UAVs is a tool with an increasing importance to perform reconnaissance and surveillance missions in dangerous environments with high risk for human life.

In this context, the development of an antenna that improves the UAV communication efficiency with the ground station was preformed. The main advantage of planar antennas is the simplicity of construction, and insertion in several surfaces, and the ability to give adequate radiation patterns. However, these antennas have low efficiency and narrow band width. The transmission line method was used to obtain a first approximation of the dimensions of the patch structure, as well as how to match the antenna to the desired impedance. The full-wave methods were used with the CST MWS simulation program.

The study began with an analysis of a single planar antenna. The choice of substrate is a key parameter in the antenna radiation characteristics, that is, factors such as the dielectric constant, the thickness of the substrate and the tangent of loss angle should be considered in the desired characteristics.

After this step begins the development of an array of patch antennas designated by P-ESPAR antenna. However the antenna array alone is not able to carry out the specifications of the initially proposed antenna, this means that it is necessary a reconfigure the antenna.

Antenna configuration of P-ESPAR takes into account various critical parameters as the choice of substrate, its dimensions, the excitation method, the selection of the varicap diodes, the capacitive reactance adjustment necessary for the required specifications, and the position of the mutual coupling diodes.

The variation characteristics of the radiation pattern of the antenna P-ESPAR that meets the operational requirements such as resonant frequency, and bandwidth are only achieved by adjusting the variable capabilities of varicap diodes. These values were adjusted after several simulations in order to find the ideal characteristics that fulfill the objectives for each configuration state of the radiation beam. The antenna P-ESPAR antenna azimuthal variations were $-25^{\circ},-20^{\circ},-15^{\circ},-10^{\circ},-5^{\circ}, 5^{\circ}, 10^{\circ}, 15^{\circ}, 20^{\circ}$ and $25^{\circ}$ in relation to its reference azimuth $\left(0^{\circ}\right)$. It was verified that the main lobe of radiation in the positive direction is symmetrical to the main lobe of radiation in the negative direction as required.

As a conclusion for the realization of this master's thesis several key studies to meet the proposed objectives were performed. This work involved the use of several simulation tools such as the CST MWS program, essential for the 
Pedro Marques, Maria Martins, António Baptista and João Paulo N. Torres/

Journal of Engineering Science and Technology Review 11 (1) (2018) 90-102

design and optimization of the P-ESPAR antenna, as well as for understanding the antenna behavior during its development. The design program Solidworks 3D CAD assisted in project design with the layout of the P-ESPAR antenna.

\section{Acknowledgement}

This work was supported by FCT, through IDMEC, under LAETA, project UID/EMS/50022/2013 and under IT, project UID/EEA/50008/2013.

This is an Open Access article distributed under the terms of the Creative Commons Attribution Licence

\section{References}

[1] M. Nogueira de Sousa, "Uso de Veículos Aéreos Não Tripulados no Sistema Tático de Guerra Eletrônica (SITAGE),” p. 12, 2008.

[2] C. J. Oliveira Ribeiro, "As Operações Militares Na Era Da Informação E Da Comunicação,” pp. 9-31, 2005.

[3] US Department of Defense, "Unmanned Aircraft Systems Roadmap 2005-2030," p. 213, 2005.

[4] J. A. Morgado and J. T. Borges de Sousa, "O PROGRAMA DE INVESTIGAÇÃO E TECNOLOGIA EM VEÍCULOS AÉREOS AUTÓNOMOS NÃO-TRIPULADOS DA ACADEMIA DA FORÇA AÉREA,” p. 16, 2015.

[5] J. P. Morgado, "Centro de Investigação da Academia da Força Aérea: Desenvolvimento \& Inovação na área dos Sistemas Aéreos Autónomos Não-Tripulados," Cid. e Def., pp. 16-21, 2015.

[6] R. Austin, Unmanned Aircraft Systems. 2010.

[7] G. B. Ronconi, T. J. Batista, and V. Merola, "The Utilization Of Unmanned Aerial Vehicles ( UAV ) For Military Action In Foreign Airspace," p. 44, 2014.

[8] L. Sun, B. Sun, J. Yuan, W. Tang, and H. Wu, "Low Profile, Quasi-
Omnidirectional, Substrate Integrated Waveguide (SIW) MultiHorn Antenna," IEEE Antennas Wirel. Propag. Lett., vol. 1225, no. c, p. 1, 2015.

[9] D. T. I. Center, "UAV Requirements and Design Consideration," p. 9, 2000.

[10] J. Leland and I. Porche III, Future Army Bandwidth Needs and Capabilities. RAND Corporation, 2004.

[11]C. A. Balanis, Antenna Theory Analysis and Design, 3rd Editio. 2005.

[12]R. Garg, P. Bhartia, I. Bahl, and A. Ittipiboon, Microstrip Antenna Design Handbook. Boston: Artech House, INC.

[13] S. Zhang, G. H. Huff, C. Cung, and J. T. Bernhard, "Three variations of a pattern reconfigurable microstrip parasitic array," Microw. Opt. Technol. Lett., pp. 369-372, 2005.

[14]J. Luther, "Microstrip Patch Electrically Steerable Parasitic Array Radiators," University of Central Florida, 2013. 